\title{
135. Über das Verhalten der Folge der Integralsysteme von gewöhnlichen Differentialgleichungen.
}

By Mitio Nagumo.

Mathematical Institute, Tokyo Imperial University.

(Rec. Sept. 19, 1928. Comm. by T. YosiE, M.I.A., Oct. 2, 1928.)

Die vorliegende Arbeit beschäftigt sich mit der Untersuchung des Verhaltens der Folge der Integralsysteme von gewöhnlichen Differentialgleichungen in normaler Form

$$
\frac{d x_{i}}{d t}=f_{i}\left\{t, x_{1}, \ldots, x_{k}, \stackrel{n}{\varphi} \varphi_{1}(t), \ldots, \stackrel{n}{\varphi}(t)\right\} \quad(i=1,2, \ldots, k),
$$

wobei die Konvergenz der Folge der Integrale von Funktionensystemen $\left\{\stackrel{\varphi}{\varphi}_{1}(t), \ldots, \varphi_{\lambda}(t)\right\}$ vorausgesetzt wird. Dabei ist die Stetigkeit der Funktionen nicht notwendig vorausgesetzt. Hier verstehen wir unter Integralsystemen dieser Differentialgleichungen mit den Anfangsbedingungen $x_{i}\left(t_{0}\right)=\xi^{\prime}{ }_{i}$ die Funktionensysteme $\left\{x_{1}(t), \ldots, x_{k}(t)\right\}$, die den Gleichungssystemen

$$
x_{i}(t)=\xi_{i}^{\prime}+\int_{t_{0}}^{t} f_{i}\left\{t, x_{1}(t), \ldots, x_{k}(t), \stackrel{n}{\varphi}(t), \ldots, \stackrel{n}{\varphi}(t)\right\} d t \quad(i=1,2, \ldots, k)
$$

genügen, wobei die Integrale im Sinne von de la Vallée-Poussin verstanden werden. Dann erhalten wir die folgenden Sätze, deren Beweise in "Japanese Journal of Mathematics" erscheinen werden. Der Kürze halber bezeichnen wir mit $\bar{B}$ den Bereich $a \leqq t \leqq b,\left|x_{i}-\xi_{i}\right| \leqq l$, $-\infty<\varphi_{\mu}<\infty$ und mit $B$ den Bereich $a \leqq t \leqq b,\left|x_{i}-\xi_{i}\right| \leqq l$.

I. Die Funktionen $F_{i}\left\{t, x_{1}, \ldots, x_{k}, \varphi_{1}, \ldots, \varphi_{\lambda}\right\}$ und ihre partiellen Ableitungen $\frac{\partial F_{i}}{\partial \varphi_{\mu}}$ und $\frac{\partial^{2} F_{i}}{\partial \varphi_{\mu} \partial \varphi_{\nu}}(\mu, \nu=1, \ldots, \lambda)$ seien in $\bar{B}$ stetig. $F_{i}$ genügen auch für $\left|\varphi_{\mu}\right| \leqq h$ in $B$ der Lipschitzbedingung

$$
\begin{gathered}
\left|F_{i}^{\prime}\left\{t, x_{1}^{*}, \ldots, x_{k}^{*}, \varphi_{1}, \ldots, \varphi_{\lambda}\right\}-F_{i}\left\{t, x_{1}, \ldots, x_{k}, \varphi_{1}, \ldots, \varphi_{\lambda}\right\}\right| \\
\leqq K_{h} \sum_{j=1}^{k}\left|x_{j}^{*}-x_{j}\right|,
\end{gathered}
$$

wobei $K_{h}$ nur von $h$ abhängige Konstante bedeutet. Es seien $\stackrel{n}{x_{i}}(t)$ und 
$x_{i}(t)$ die in einem Teilbereich von $B$ liegenden Integralsysteme der Differentialgleichungen

$$
\frac{d x_{i}}{d t}=F_{i}\left\{t, x_{1}, \ldots, x_{k}, \stackrel{n}{\varphi}(t), \ldots, \stackrel{n}{\varphi}(t)\right\}
$$

bzw.

$$
\frac{d x_{i}}{d t}=F_{i}\left\{t, x_{1}, \ldots, x_{k}, \varphi_{1}(t), \ldots, \varphi_{\lambda}(t)\right\}
$$

mit den Anfangsbedingungen $\stackrel{n}{x_{i}}\left(t_{0}\right)=\stackrel{n}{\xi_{i}}$ bzw. $x_{i}\left(t_{0}\right)=\xi_{i}$, wobei $\varphi_{\mu}(t)$ und $\stackrel{\imath}{\varphi}_{\mu}(t)$ in $\langle a, b\rangle$ summierbare Funktionen sind. Dann erhält $\operatorname{man}$ :

Dafür, dass nur aus den Relationen

und

$$
\left|\varphi_{\mu}(t)\right| \leqq h, \quad\left|\varphi_{\mu}(t)\right| \leqq h, \lim _{n \rightarrow \infty} \xi_{i}^{n}=\xi_{i}^{\prime}
$$

$$
\lim _{n \rightarrow \infty} \int_{t_{0}}^{t} \varphi_{\mu}^{n}(t) d t=\int_{t_{0}}^{t} \varphi_{\mu}(t) d t
$$

stets die Gleichungen

$$
\lim _{n \rightarrow \infty} x_{i}^{n}(t)=x_{i}(t) \quad(i=1,2, \ldots, k)
$$

folgen, wobei $h$ eine beliebige feste Konstante und $\left(t_{0}, \xi^{\prime}{ }_{1}, \ldots, \xi^{\prime}{ }_{k}\right)$ einen beliebigen inneren Punkt von $B$ bedeutet, ist es notwendig und hinreichend, dass $F_{i}$ in bezug auf $\left(\varphi_{1}, \varphi_{2}, \ldots, \varphi_{\lambda}\right)$ linear sind.

II. Die Funktionen $F_{i}$ und ihre partiellen Ableitungen $\frac{\partial F_{i}}{\partial \varphi_{\mu}}$ seien in $\bar{B}$ stetig. $\quad F_{i}$ genügen auch der Bedingung

$$
\begin{gathered}
\left|F_{i}\left\{t, x_{1}{ }^{*}, \ldots, x_{k}{ }^{*}, \varphi_{1}, \ldots, \varphi_{\lambda}\right\}-F_{i}\left\{t, x_{1}, \ldots, x_{k}, \varphi_{1}, \ldots, \varphi_{\lambda}\right\}\right| \\
\leqq K\left(\varphi_{1}, \ldots, \varphi_{\lambda}\right) \sum_{j=1}^{k}\left|x_{j}{ }^{*}-x_{j}\right|
\end{gathered}
$$

wobei $K$ eine nicht negative stetige Funktion von $\left(\varphi_{1}, \ldots, \varphi_{\lambda}\right)$ bedeutet. Es seien $x_{i}(t)$ und $x_{i}(t)$ die in $B$ liegenden Integralsysteme der Differentialgleichungen

$$
\frac{d x_{i}}{d t}=F_{i}\left\{t, x_{1}, \ldots, x_{k}, \stackrel{n}{\varphi}(t), \ldots, \stackrel{n}{\varphi} \varphi_{\lambda}(t)\right\}
$$

bzw.

$$
\frac{d x_{i}}{d t}=F_{i}\left\{t, x_{1}, \ldots, x_{k}, \varphi_{1}(t), \ldots, \varphi_{\lambda}(t)\right\}
$$

mit den Anfangsbedingungen $\stackrel{n}{x}_{i}\left(t_{0}\right)=\stackrel{n}{\xi_{i}}$ bzw. $x_{i}\left(t_{0}\right)=\xi_{i}$, wobei $\stackrel{n}{\varphi}_{\mu}(t)$ in 
$\langle a, b\rangle$ summierbare und $\varphi_{\mu}(t)$ in $\langle a, b\rangle$ stetige Funktionen sind. Weiter definieren wir

$$
\begin{aligned}
& \mathscr{G}_{i}\left\{t, x_{1}, \ldots, x_{k} ; \varphi_{1}, \ldots, \varphi_{\lambda} ; \varphi_{1}^{*}, \ldots, \varphi_{\lambda}^{*}\right\} \\
& \equiv F_{i}\left\{t, x_{1}, \ldots, x_{k}, \varphi_{1}^{*}, \ldots, \varphi_{\lambda}^{*}\right\}-F_{i}\left\{t, x_{1}, \ldots, x_{k}, \varphi_{1}, \ldots, \varphi_{\lambda}\right\} \\
& -\sum_{\mu=1}^{\lambda}\left(\varphi_{\mu}^{*}-\varphi_{\mu}\right) \frac{\partial F_{i}}{\partial \varphi_{\mu}}\left\{t, x_{1}, \ldots, x_{k}, \varphi_{1}, \ldots, \varphi_{\lambda}\right\} \text {. } \\
& \left|\mathscr{F}_{i}\left\{t, x_{1}, \ldots, x_{k} ; \varphi_{1}(t) ; \ldots, \varphi_{\lambda}(t) ; \varphi_{1}^{*}, \ldots, \varphi_{\lambda}^{*}\right\}\right| \\
& \leqq \alpha_{\mathscr{O}}\left\{t, x_{1}, \ldots, x_{k} ; \varphi_{1}(t), \ldots, \varphi_{\lambda}(t) ; \varphi_{1}^{*}, \ldots, \varphi_{\lambda}^{*}\right\}(i=1,2, \ldots, k)
\end{aligned}
$$

in $B$ für alle $\varphi^{*}$, wobei $\alpha$ eine positive Konstante ist,

$$
\begin{gathered}
\int_{t_{0}}^{t}\left|\varphi_{\mu}(t)\right| d t \leqq H \text { für alle } n(H: \text { eine beliebige feste Konstante), } \\
\lim _{n \rightarrow \infty}{\stackrel{n}{\xi_{i}}}^{2}=\xi_{i}^{\prime}, \quad \int_{t_{0}}^{t} K\left(\stackrel{n}{\varphi}_{1}(t), \ldots, \stackrel{n}{\varphi}_{\lambda}(t)\right) d t \leqq L,
\end{gathered}
$$

wobei die Konstante $L$ die Bedingung $k \alpha L e^{k L} \leqq 1$ erfüllt, und im Sinne der gleichmässigen Konvergenz

gelten dann die Ungleichungen

$$
\lim _{n \rightarrow \infty} \int_{t_{0}}^{t} \varphi_{\mu}(t) d t=\int_{t_{0}}^{t} \varphi_{\mu}(t) d t \quad\left(t \leqq t_{0}\right),
$$

$$
\lim _{n \rightarrow \infty} x_{1}(t) \geqq x_{1}(t) \quad \text { fur } t \geqq t_{0} \text {. }
$$

III. Es sei eine Folge der Differentialgleichungssysteme

$$
\text { (A) }
$$

$$
\begin{aligned}
\frac{d x_{i}}{d t} & =f_{i}\left\{t, x_{1}, \ldots, x_{k}, n_{1}(t), \ldots, \stackrel{n}{\varphi}_{\lambda}(t)\right\} \\
& +\sum_{\mu=1}^{\lambda} \varphi_{\mu}^{n}(t) g_{i \mu}\left\{t, x_{1}, \ldots, x_{k}\right\} \quad(i=1,2, \ldots, k)
\end{aligned}
$$

vorgelegt, wobei $f_{i}$ in $\bar{B}$ beschränkt und $g_{i \mu}$ samt ihren partiellen Ableitungen $\frac{\partial g_{i \mu}}{\partial x_{j}}(i, j=1,2, \ldots, k)(\mu=1, \ldots, \lambda)$ in $B$ stetig sind. Die Funktionen $\stackrel{n}{\varphi}_{\mu}(t)$ seien im Intervalle $\left\langle t_{0}-\delta_{n}, t_{0}+\delta_{n}\right\rangle$ summierbar. $x_{i}(t)$ seien die für $t_{0}-\grave{o}_{n} \leqq t<t_{0}+\partial_{n}$ in $B$ liegenden Integralsysteme der Differentialgleichungen (A) mit den Anfangsbedingungen ${ }_{i}^{n}\left(t_{0}-\hat{\sigma}_{n}\right)=\stackrel{n}{\xi}_{i}$. Dann haben wir : 
Dafür, dass nur aus den Relationen

$$
\begin{gathered}
\int_{t_{0}-\delta n}^{t_{0}+\delta n}\left|\stackrel{\varphi}{\varphi}_{\mu}(t)\right| d t \leqq H, \quad \lim _{n \rightarrow \infty} \delta_{n}=0, \quad \lim _{n \rightarrow \infty} \stackrel{n}{\xi}_{i}=\xi_{i}^{\prime} \\
\lim _{n \rightarrow \infty} \int_{t_{0}-\delta n}^{t_{0}+\delta n} \varphi_{\mu}(t) d t=\Phi_{\mu},
\end{gathered}
$$

und

wobei $\left(t_{0}, \xi_{1}^{\prime}, \ldots, \xi_{k}^{\prime}\right)$ einen beliebigen inneren Punkt von $B$ bedeutet, stets die Gleichungen

$$
\lim _{n \rightarrow \infty} x_{i}\left(t_{0}+\delta_{n}\right)=X_{i}\left(\Phi_{1}, \ldots, \Phi_{\lambda} ; t_{0}, \xi_{1}^{\prime}, \ldots, \xi_{k}^{\prime}\right)
$$

folgen $^{1)}$, dabei $X_{i}$ nur von $\Phi_{1}, \ldots, \Phi_{\lambda}, t_{0}, \xi^{\prime}{ }_{1}, \ldots, \xi^{\prime}{ }_{k}$ abhängige Funktionen bedeuten, ist es notwendig und hinreichend, dass die Funktionen $g_{i \mu}(i=1,2, \ldots, k)$ in $B$ der Bedingung

$$
\sum_{j=1}^{k} \frac{\partial g_{i \mu}}{\partial x_{j}} \cdot g_{j \nu}=\sum_{j=1}^{k} \frac{\partial g_{i v}}{\partial x_{j}} g_{j, \iota} \quad(\mu, \nu=1,2, \ldots, \lambda)
$$

genügen.

1) Das ist für $\gamma=1$ stets der Fall. 